\title{
A set valued characterization of ISDS Lyapunov functions
}

\author{
Lars Grüne and Patrick Saint-Pierre
}

\begin{abstract}
We use set valued analysis techniques in order to characterize Lyapunov functions for the input-to-state dynamical stability (ISDS) property, a quantitatively sharper but qualitatively equivalent variant of the well known inputto-state stability (ISS) property. We show that the epigraphs of minimal ISDS Lyapunov functions are invariance kernels of a suitable augmented differential inclusion. This identity provides theoretical insight into local ISDS properties and yields a basis for a numerical approximation of ISDS and ISS Lyapunov functions via set oriented numerical methods.
\end{abstract}

\section{INTRODUCTION}

One of the key concepts in nonlinear stability theory for perturbed systems is the input-to-state stability property (ISS), introduced by E.D. Sontag in 1989 [12] and further investigated in, e.g., [7], [13], [15]. The ISS property can be seen as a generalization of the asymptotic stability property to perturbed systems of the type $\dot{x}(t)=f(x(t), w(t))$ and demands that each trajectory $\varphi$ satisfies the inequality

$$
\|\varphi(t, x, w)\| \leq \max \left\{\beta(\|x\|, t), \gamma\left(\|w\|_{\infty}\right)\right\}
$$

for suitable, so called, comparison functions $\beta \in \mathcal{K} \mathcal{L}$ and $\gamma \in$ $\mathcal{K}_{\infty} \cdot{ }^{1}$ For a survey about the ISS property and its applications in nonlinear systems theory we refer to the survey [14] and the references therin.

One of the early important results about the ISS property was the observation that it can be characterized by a suitable Lyapunov function, see [15].More precisely, the ISS property is equivalent to the existence of a continuously differentiable function $V: \mathbb{R}^{n} \rightarrow \mathbb{R}$ satisfying the bounds

$$
\|x\| \leq V(x) \leq \sigma(\|x\|)
$$

for some $\sigma \in \mathcal{K}_{\infty}$, and the decaying property

$$
\inf _{\gamma(\|w\|) \leq V(x)} D V(x) f(x, w) \leq-g(V(x))
$$

for some $g: \mathbb{R}_{0}^{+} \rightarrow \mathbb{R}_{0}^{+}$with $g(r)>0$ for $r>0$. This Lyapunov function characterization comes in different variants, and the fact that we prefer this particular form lies in the fact that integrating (3) for some perturbation function $w$ and using (2) one obtains (1) with $\gamma$ from (3) and $\beta(r, t)=\mu(\sigma(r), t)$ where $\mu$ is the solution of the initial value problem $\dot{\mu}=-g(\mu), \mu(0)=r$. Hence, the functions

This research was done while the first author was a professeur invité at the Université Paris IX Dauphine

L. Grüne is with Mathematisches Institut, Universität Bayreuth, 95440 Bayreuth, Germany, lars.gruene@uni-bayreuth.de

P. Saint-Pierre is with Centre de recherche Viabilité, Jeux, Contrôle, Université Paris IX Dauphine, Place du Maréchal de Lattre de Tassigny, 75775 Paris Cedex, France, Patrick.Saint-Pierre@dauphine.fr

${ }^{1}$ These function classes, nowadays standard in nonlinear stability theory, are defined in Section II. $\sigma, \gamma$ and $\mu$ from $V$ are directly related to the comparison functions $\beta$ and $\gamma$ in the ISS estimate (1).

A more careful investigation of this relation reveals that the existence of $V$ with (2), (3) implies a slightly stronger property than ISS, namely the input-to-state dynamical stability property (ISDS) introduced in [4, Chapter 3] and [5] (see also [6]). The ISDS property, which will be precisely defined in Definition 2.1, below, is qualitatively equivalent to ISS (see [4, Proposition 3.4.4(ii)]) but, due to its tighter quantitative relation to $V$, more suitable for a Lyapunov function based analysis. Hence, in this paper we will work with this ISDS property which we will use in a rather general version by considering arbitrary compact sets $A$ instead of the origin, and by allowing that ISDS only holds on a subset $B \subseteq \mathbb{R}^{n}$ instead of the whole $\mathbb{R}^{n}$.

This paper deals with the characterization of the ISDS property and ISDS Lyapunov functions using set valued techniques. More precisely, to our $n$-dimensional perturbed system we associate an augmented $n+1$-dimensional differential inclusion with solutions $\psi$, where the additional dimension represents the value of the Lyapunov function $V$. Via this inclusion we obtain a characterization of $V$ via the invariance kernel $\operatorname{Inv}_{\psi}(D)$ of a suitable set $D$. In particular, we are able to give a necessary and sufficient condition on the shape of $\operatorname{Inv}_{\psi}(D)$ being equivalent to the ISDS property. Furthermore, the invariance kernel $\operatorname{Inv}_{\psi}(D)$ characterizes the minimal ISDS Lyapunov function by means of its epigraph, provided that ISDS holds. However, even when ISDS does not hold the set $\operatorname{Inv}_{\psi}(D)$ may contain useful information. If ISDS does not hold for some perturbation range $W$, then it may still hold for a suitably restricted perturbation range $\widetilde{W}$. It turns out that the invariance kernel $\operatorname{Inv}_{\psi}(D)$ for the unrestricted perturbation set $W$ can be used in order to determine whether this is the case, and if so, then $\operatorname{Inv}_{\psi}(D)$ gives a precise estimate about the size of the maximal restricted perturbation range $\widetilde{W}$ for which ISDS holds.

The contribution of these results is twofold. First, our results give additional insight into the ISDS (and thus the ISS) property and the respective Lyapunov functions. In particular, our second result characterizes the situation where ISDS is lost due to a too large set of perturbations, a topic which was recently investigated in [3] using a controllability analysis. Second, since invariance kernels are computable by set valued numerical algorithms, our characterization leads to a numerical approach for computing ISDS Lyapunov functions for which - to the best of our knowledge no other numerically feasible representation is available until now. It goes without saying that the numerical effort of this approach is rather high such that our method 
is only applicable to moderately complex systems of low dimensions, but this is due to the inherent complexity of the problem, taking into account that the computation of nonlinear Lyapunov functions is a difficult task even for unperturbed systems. This numerical approach bears some similarities with a recently developed dynamic programming method for the computation of ISS comparison functions [8], with the difference that here Lyapunov functions are computed while in [8] the comparison functions (or gains) are obtained.

This paper is organized as follows. In the ensuing Section II we summarize the necessary background information on the ISDS property. In Section III we state and prove our first main result on the representation of ISDS Lyapunov functions $V$ via invariance kernels. Section IV gives necessary and sufficient conditions for ISDS using a suitably restricted perturbation range. Finally, in Section V, we show some examples.

\section{SETUP AND PRELIMINARIES}

We consider perturbed nonlinear systems of the form

$$
\dot{x}(t)=f(x(t), w(t))
$$

with $x \in \mathbb{R}^{n}$, and $w \in \mathcal{W}:=L_{\infty}(\mathbb{R}, W)$ for some $W \subseteq \mathbb{R}^{l}$. We assume that $f$ is continuous and Lipschitz in $x$ uniformly for $w$ in a compact set. We denote the solutions with $\varphi(t, x, w)$.

For a compact set $A \subset \mathbb{R}^{n}$ we denote the Euclidean distance to $A$ by $d_{A}$.

We define the comparison function classes

$$
\begin{aligned}
\mathcal{K}:= & \left\{\alpha: \mathbb{R}_{0}^{+} \rightarrow \mathbb{R}_{0}^{+} \mid \alpha\right. \text { is continuous and strictly } \\
& \text { increasing with } \alpha(0)=0\} \\
\mathcal{K})_{\infty}:= & \{\alpha \in \mathcal{K} \mid \alpha \text { is unbounded }\} \\
\mathcal{L}:= & \left\{\alpha: \mathbb{R}_{0}^{+} \rightarrow \mathbb{R}_{0}^{+} \mid \alpha\right. \text { is continuous and strictly } \\
& \text { decreasing with } \left.\lim _{t \rightarrow \infty} \alpha(t)=0\right\} \\
\mathcal{K} \mathcal{L}:= & \left\{\beta: \mathbb{R}_{0}^{+} \times \mathbb{R}_{0}^{+} \rightarrow \mathbb{R}_{0}^{+} \mid \beta\right. \text { is continuous, } \\
& \beta(\cdot, t) \in \mathcal{K}, \beta(r, \cdot) \in \mathcal{L} \text { for all } t, r \geq 0\} \\
\mathcal{K} \mathcal{L} \mathcal{D}:= & \{\mu \in \mathcal{K} \mathcal{L} \mid \mu(r, 0)=r, \\
& \mu(r, t+s)=\mu(\mu(r, t), s) \text { for all } r, t, s \geq 0\}
\end{aligned}
$$

The first four classes are standard in nonlinear stability theory while the last class $\mathcal{K} \mathcal{L} \mathcal{D}$ of "dynamical" $\mathcal{K} \mathcal{L}$ functions was introduced in [4] in order to formalize the specific form of $\mathcal{K} \mathcal{L}$ functions $\beta(r, t)=\mu(\sigma(r), t)$ originating from the integration of a Lyapunov function, cf. the introduction.

Using these functions we can now define the ISDS property.

Definition 2.1: The set $A$ is called input-to-state dynamically stable (ISDS) on some open neighborhood $B$ of $A$, if for suitable $\mu \in \mathcal{K} \mathcal{L D}$ and $\sigma, \gamma \in \mathcal{K}_{\infty}$ and all $x \in B$, all $w \in \mathcal{W}$ and all $t \geq 0$ the inequality

$$
d_{A}(\varphi(t, x, w)) \leq \max \left\{\mu\left(\sigma\left(d_{A}(x), t\right), \nu(w, t)\right\}\right.
$$

holds with

$$
\nu(w, t):=\operatorname{ess}_{\tau \in[0, t]} \mu(\gamma(\|w(\tau)\|), t-\tau) .
$$

We call $A$ globally ISDS if this property holds with $B=\mathbb{R}^{n}$.

The most important feature of the ISDS property is its quantitative characterization by an ISDS Lyapunov function. If $B \neq \mathbb{R}^{n}$ then for its definition we need the reachable set $\mathcal{R}_{\varphi, W}(B)$ of a set $B$ under $\varphi$, defined by

$$
\mathcal{R}_{\varphi, W}(B):=\bigcup_{w \in L_{\infty}(\mathbb{R}, W), x \in B, t \in\left[0, T_{\max }(x, w)\right)}\{\varphi(t, x, w)\},
$$

where $T_{\max }(x, w)$ denotes the upper bound of the existence interval of the solution $\varphi(t, x, w)$.

Definition 2.2: A function $V: \mathcal{R}_{\varphi, W}(B) \rightarrow \mathbb{R}$ is called an ISDS Lyapunov function, if it satisfies the inequalities

$$
\begin{aligned}
& V(x) \geq d_{A}(x) \quad \text { for all } x \in \mathcal{R}_{\varphi, W}(B) \\
& V(x) \leq \sigma\left(d_{A}(x)\right) \quad \text { for all } x \in B
\end{aligned}
$$

and

$$
V(\varphi(t, x, w)) \leq \max \{\mu(V(x), t), \nu(w, t)\}
$$

for all $x \in \mathbb{R}^{n}, w \in \mathcal{W}$ and $t \geq 0$ with $\nu$ from (6).

It is easily seen that the existence of $V$ meeting Definition 2.2 implies ISDS with the same comparison functions. The converse is also true but much less trivial to prove, cf. [4, Theorem 3.5.3] or [5, Theorem 4] $]^{2}$. Thus, an ISDS Lyapunov function for given comparison functions $\mu, \sigma, \gamma$ exists if and only if the set $A$ is ISDS for these comparison functions and the ISDS property admits a precise quantitative charactarization by ISDS Lyapunov functions.

In the remainder of this paper we will always assume that the function $\mu \in \mathcal{K} \mathcal{L} \mathcal{D}$ satisfies the differential equation

$$
\frac{d}{d t} \mu(r, t)=-g(\mu(r, t))
$$

for some Lipschitz continuous $g: \mathbb{R} \rightarrow \mathbb{R}$ with $g(r)>0$ for $r>0$. By [4, Proposition B.2.3] this can be assumed without loss of generality, more precisely, for any given $\tilde{\mu} \in \mathcal{K} \mathcal{L} \mathcal{D}$ we find $\mu \in \mathcal{K} \mathcal{L} \mathcal{D}$ arbitrarily close to $\tilde{\nu}$ satisfying (9).

Remark 2.3: If the function $V$ from Definition 2.2 is smooth and $\mu$ satisfies (9), then (8) is equivalent to the infinitesimal inequality (3), see [5, Lemma 15]. Even if $V$ is not smooth one can use this infinitesimal characterization, when interpreted in the viscosity solution sense, see [4, Proposition 3.5.6] for details. In this paper, we will work directly with (8), thus avoiding the use of nonsmooth differential calculus.

\section{AN INVARIANCE KERNEL REPRESENTATION}

Fixing two functions $\gamma \in \mathcal{K}_{\infty}$ and $\mu \in \mathcal{K} \mathcal{L} \mathcal{D}$ satisfying (9), to our perturbed system (4) we associate the $n+1-$ dimensional differential inclusion

$$
\begin{aligned}
\dot{x}(t) & \in f(x(t), W(y(t))) \\
\dot{y}(t) & =-g(y(t)) \\
\text { with } W(y) & =\{w \in W \mid \gamma(\|w\|) \leq y\}
\end{aligned}
$$

${ }^{2}$ In fact, in [5] only the special case $A=\{0\}$ and $B=\mathbb{R}^{n}$ is treated, but the proof easily carries over to our more general setting. 
and $y \in \mathbb{R}_{0}^{+}$. We denote the solutions by $\psi(t, x, y)$, by $\psi(t, z)$ for $z=(x, y) \in \mathbb{R}^{n+1}$ or simply by $\psi(t)$, if there is no ambiguity. We will frequently use the decomposition $\psi(t)=$ $\left(\psi_{x}(t), \psi_{y}(t)\right)$ with $\psi_{x}(t) \in \mathbb{R}^{n}$ and $\psi_{y}(t) \in \mathbb{R}$. We assume that the right hand side of this differential inclusion and the map $y \rightsquigarrow W(y)$ are Lipschitz set valued maps, which holds, e.g., if $W$ is a star shaped set and $\gamma^{-1}$ is Lipschitz, which can be assumed without loss of generality.

The following sets will be crucial for our analysis.

For a subset $D \subset \mathbb{R}^{n+1}$ and a differential inclusion with solutions denoted by $\psi$ we define its (forward) invariance kernel as

$\operatorname{Inv}_{\psi}(D):=\left\{\begin{array}{l|l}z \in D & \begin{array}{l}\psi(t, z) \in D \text { for all solutions } \\ \psi \text { of }(10) \text { and all } t \geq 0\end{array}\end{array}\right\}$.

For an extended real valued function $G: \mathbb{R}^{n} \rightarrow \mathbb{R} \cup\{\infty\}$ we define its epigraph $\operatorname{Epi}(G) \subset \mathbb{R}^{n+1}$ by

$$
\operatorname{Epi}(G):=\left\{(x, y) \in \mathbb{R}^{n+1} \mid y \geq G(x)\right\} .
$$

For a set $B \subseteq \mathbb{R}^{n}$ we define

$$
\operatorname{Epi}\left(\left.G\right|_{B}\right):=\operatorname{Epi}(G) \cap(B \times \mathbb{R}) .
$$

Since ISDS Lyapunov functions are in general only defined on subsets $C \subset \mathbb{R}^{n}$ we extend them to $\mathbb{R}^{n}$ by setting $V(x)=\infty$ for $x \notin C$ and define $\operatorname{Dom}(V):=\{x \in$ $\left.\mathbb{R}^{n} \mid V(x)<\infty\right\}$.

The set which we are interested in is the invariance kernel $\operatorname{Inv}_{\psi}(D)$ of the set

$$
D:=\operatorname{Epi}\left(d_{A}\right)=\left\{(x, y) \in \mathbb{R}^{n+1} \mid y \geq d_{A}(x)\right\} .
$$

More precisely, we will use the largest epigraph contained in $\operatorname{Inv}_{\psi}(D)$. For this purpose, for a given closed set $E \subset$ $\mathbb{R}^{n+1}$ we define the set

$$
\mathcal{M}(E):=\{(x, y) \in E \mid(x, z) \in E \text { for all } z \geq y\} .
$$

The set $\mathcal{M}(E)$ is the largest subset of $E$ which can be written as an epigraph of a function $G: \mathbb{R}^{n} \rightarrow \mathbb{R} \cup\{\infty\}$.

Using these concepts we can now describe the relation between ISDS Lyapunov functions and suitable invariance kernels.

Theorem 3.1: Consider the perturbed system (4) and the differential inclusion (10). Consider a compact set $A \subset \mathbb{R}^{n}$, an open neighborhood $B \subseteq \mathbb{R}^{n}$ of $A$ and the set $D$ from (11). Then the following assertions hold:

(i) Each ISDS Lyapunov function $V: \mathbb{R}^{n} \rightarrow \mathbb{R}$ satisfies

$$
\operatorname{Epi}(V) \subseteq \mathcal{M}\left(\operatorname{Inv}_{\psi}(D)\right) .
$$

(ii) If there exists a function $\sigma \in \mathcal{K}_{\infty}$ such that

$$
\operatorname{Epi}\left(\left.\sigma\left(d_{A}\right)\right|_{B}\right) \subseteq \operatorname{Inv}_{\psi}(D)
$$

holds, then there exists an ISDS Lyapunov function $V$ : $\mathbb{R}^{n} \rightarrow \mathbb{R}$ with

$$
B \subseteq \operatorname{Dom}(V) \text { and } \operatorname{Epi}(V)=\mathcal{M}\left(\operatorname{Inv}_{\psi}(D)\right) .
$$

In particular, this $V$ is the minimal ISDS Lyapunov function for (4) in the sense that $V(x) \leq \widetilde{V}(x)$ holds for all $x \in$
$\operatorname{Dom}(V)$ and all other ISDS Lyapunov functions $\widetilde{V}$ for the comparison functions $\mu$ and $\gamma$.

(iii) The set $A$ is ISDS with neighborhood $B$ if and only if (12) holds for some function $\sigma \in \mathcal{K}_{\infty}$.

Proof: By [5, Lemma 13] a function $V: \mathcal{R}(B) \rightarrow \mathbb{R}$ satisfies (8) if and only if it satisfies

$$
\begin{aligned}
& V(\varphi(t, x, w)) \leq \mu(y, t) \text { for all } x \in B, \text { all } t \geq 0 \\
& \text { all } y \geq V(x) \text { and all } w \in \mathcal{W} \text { with } \\
& \gamma(\|w(\tau)\|) \leq \mu(y, \tau) \text { for almost all } \tau \in[0, t] .
\end{aligned}
$$

For the sake of completeness we give the proof of the equivalence $(8) \Leftrightarrow(13)$.

Assume (8) and $w \in \mathcal{W}$ is such that $\gamma(\|w(\tau)\|) \leq \mu(y, \tau)$ holds for almost all $\tau \in[0, t]$. Then the definition of $\nu$ in (6) implies $\nu(w, t) \leq \mu(y, t)$, thus (8) immediately implies (13).

Conversely, assume (13) and consider $w \in \mathcal{W}, x \in B$ and $t \geq 0$. Set $y=\max \{V(x), \mu(\nu(w, t),-t)\}$, which by (6) implies $\gamma(\|w(\tau)\|) \leq \mu(y, \tau)$ for almost all $\tau \in[0, t]$, hence (13) implies $V(\varphi(t, x, w)) \leq \mu(y, t)$. Now by the choice of $y$ either $y=V(x)$ or $\mu(y, t)=\nu(w, t)$ holds. In the first case, from (13) we obtain $V(\varphi(t, x, w)) \leq$ $\mu(y, t)=\mu(V(x), t)$ while in the second case we obtain $V(\varphi(t, x, w)) \leq \mu(y, t)=\nu(w, t)$. In both cases, (8) follows.

Using this equivalence we now turn to the proof of the theorem.

(i) Let $(x, y) \in \operatorname{Epi}(V)$ and let $\psi(t)=\psi(t, x, y)$ be a solution of the differential inclusion (10). We have to prove that $(x, y) \in \operatorname{Inv}_{\psi}(D)$, i.e. $\psi(t) \in D$ for all $t \geq 0$. Writing $\psi=\left(\psi_{x}, \psi_{y}\right)$ this amounts to showing $d_{A}\left(\psi_{x}(t)\right) \leq \psi_{y}(t)$ for all $t \geq 0$. From Filippov's Lemma (see [1] or [9, p. 267]) we find a function $w(t)$ with $w(t) \in W\left(\psi_{y}(t)\right)$ for almost all $t \geq 0$ such that $\psi_{x}$ solves

$$
\frac{d}{d t} \psi_{x}(t)=f(\psi(x(t)), w(t)) .
$$

Since $\psi_{y}(t)=\mu(y, t)$ we obtain that $\gamma(\|w(\tau)\|) \leq \mu(y, \tau)$ for almost all $\tau \geq 0$. Thus from (13) we can conclude $V\left(\psi_{x}(t)\right) \leq \mu(y, t)$ which implies

$$
d_{A}\left(\psi_{x}(t)\right) \leq V\left(\psi_{x}(t)\right) \leq \mu(y, t)=\psi_{y}(t)
$$

i.e., $\psi(t) \in D$ and thus $(x, y) \in \operatorname{Inv}_{\psi}(D)$.

(ii) We show that the function $V(x)$ defined by

$$
V(x):=\inf \left\{y \geq 0 \mid(x, y) \in \mathcal{M}\left(\operatorname{Inv}_{\psi}(D)\right)\right\}
$$

(with the convention $\inf \emptyset=\infty$ ) is an ISDS Lyapunov function. Clearly, the inequalities (7) follow immediately from the construction and (12). It remains to show (8) for $x \in B$ which we do by verifying (13) for $x \in \operatorname{Dom}(V)$. Consider $t \geq 0, x \in \operatorname{Dom}(V), w \in \mathcal{W}$. Then we find $y \geq 0$ with $(x, y) \in \operatorname{Inv}_{\psi}(D)$ such that $\gamma(\|w(\tau)\|) \leq \mu(y, \tau)$ holds for almost all $\tau \in[0, t]$. The choice of $y$ implies that $w(\tau) \in W(\mu(y, \tau))$ for almost all $\tau \in[0, t]$, hence $\psi(\tau):=(\varphi(\tau, x, w), \mu(y, \tau))$ is a solution of the inclusion on $[0, \tau]$. Since $\operatorname{Inv}_{\psi}(D)$ is forward invariant we obtain $\psi(\tau) \in$ 
$\operatorname{Inv}_{\psi}(D)$ for all $\tau \in[0, t]$, in particular $\psi(t) \in \operatorname{Inv}_{\psi}(D)$. From the definition of $V$ we obtain

$$
V(\varphi(t, x, w)) \leq \mu(y, t),
$$

i.e. (13) which shows that $V$ is an ISDS Lyapunov function.

The fact that this $V$ is minimal follows immediately from (i), because each ISDS Lyapunov function $V$ satisfies $\operatorname{Epi}(V) \subseteq \mathcal{M}\left(\operatorname{Inv}_{\psi}(D)\right)$, hence the one satisfying $\operatorname{Epi}(V)=$ $\mathcal{M}\left(\operatorname{Inv}_{\psi}(D)\right)$ must be the minimal one.

(iii) If the condition (12) holds, then by (ii) we obtain the existence of an ISDS Lyapunov function with $\operatorname{Dom}(V) \supseteq B$, hence ISDS on $B$. Conversely, if ISDS holds, then by [5, Theorem 4] there exists an ISDS Lyapunov function on $B$, thus from (i) we can conclude that $\operatorname{Inv}_{\psi}(D)$ contains an epigraph containing the points $(x, V(x))$ for $x \in B$, thus for $\sigma \in \mathcal{K}_{\infty}$ from (7) $\operatorname{Inv}_{\psi}(D)$ contains the points $\left(x, \sigma\left(d_{A}(x)\right)\right)$ for $x \in B$. Hence (12) follows.

Remark 3.2: The condition (12) involving $\sigma$ implies that $\mathcal{M}\left(\operatorname{Inv}_{\psi}(D)\right)$ is not empty, that $V$ is continuous at $\partial V$ and that $V$ is bounded on compact sets. Thus, it guarantees the existence of a function $V$ with $\operatorname{Epi}(V)=\mathcal{M}\left(\operatorname{Inv}_{\psi}(D)\right)$ as well as some regularity properties of $V$. The inequality (8) is then a consequence of the structure of the differential inclusion (10).

A particular nice situation occurs when $\operatorname{Inv}_{\psi}(D)=$ $\mathcal{M}\left(\operatorname{Inv}_{\psi}(D)\right)$. In this cas we can state the following corollary.

Corollary 3.3: Consider the perturbed system (4) and the differential inclusion (10). Consider a compact set $A \subset \mathbb{R}^{n}$, an open neighborhood $B \subseteq \mathbb{R}^{n}$ of $A$ and the set $D$ from (11).

Assume that there exists a function $V: \mathbb{R}^{n} \rightarrow \mathbb{R} \cup\{\infty\}$ and a function $\sigma \in \mathcal{K}_{\infty}$ such that

$$
\operatorname{Epi}\left(\left.\sigma\left(d_{A}\right)\right|_{B}\right) \subseteq \operatorname{Epi}(V)=\operatorname{Inv}_{\psi}(D)
$$

holds. Then $V$ is an ISDS Lyapunov function on $B$ and, in particular, the set $A$ is ISDS with neighborhood $B$.

Proof: Follows immediately from Theorem 3.1 (ii).

Note that the equality $\mathcal{M}\left(\operatorname{Inv}_{\psi}(D)\right)=\operatorname{Inv}_{\psi}(D)$ need not hold, even if $\mathcal{M}\left(\operatorname{Inv}_{\psi}(D)\right) \neq \emptyset$, see Example 18, below. Hence, Corollary 3.3 indeed describes a special situation which can, hovewer, be observed for many systems.

\section{ISDS FOR RESTRICTED PERTURBATION RANGE}

Observe that $\operatorname{Inv}_{\psi}(D)$ for $D=\operatorname{Epi}\left(d_{A}\right)$ may be empty, even when no perturbations are present, e.g., when the set $A$ is not forward invariant, like the set $A=\{1\}$ for the simple 1d system $\dot{x}(t)=x(t)$. Whenever $A$ is forward invariant under $\varphi$ for $w \equiv 0$ is is easily seen that $\operatorname{Inv}_{\psi}(D)$ contains at least the set $A \times\{0\}$.

By Theorem 3.1 (iii), both $\operatorname{Inv}_{\psi}(D)=\emptyset$ and $\operatorname{Inv}_{\psi}(D)=$ $A \times\{0\}$ imply that ISDS does not hold. However, the converse is not true, i.e., if ISDS does not hold then $\operatorname{Inv}_{\psi}(D)$ might still be nonempty and strictly larger than $A \times\{0\}$. As an example, consider the $1 \mathrm{~d}$ system

$$
\dot{x}(t)=-x(t)(1-2 x(t))+w(t) .
$$

We have computed the invariance kernel of $D$ for $A=$ $\{0\}$ (i.e., $d_{A}=\|\cdot\|$ is the Euclidean norm), $\mu(r, t)=$ $e^{-t / 10} r$ (i.e., $\left.d / d t \mu(r, t)=-1 / 10 \mu(r, t)\right), \gamma(r)=2 r$ (i.e., $\left.\gamma^{-1}(r)=r / 2\right)$, and $W=\mathbb{R}$, using the numerical algorithms described in [11], [2]. Figure 1 shows the numerically computed result.

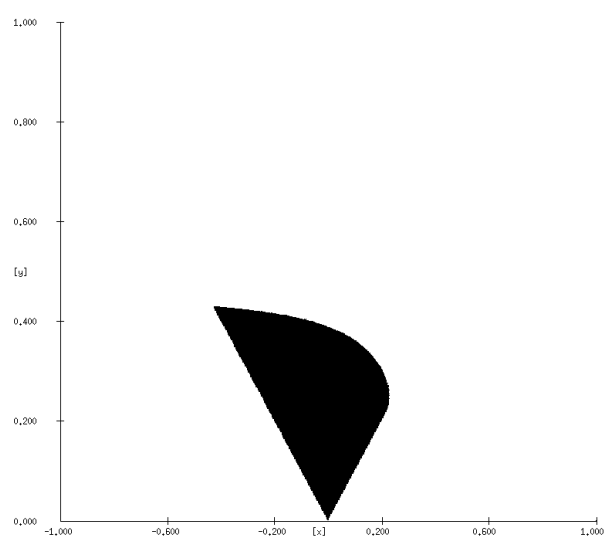

Fig. 1. Numerically determined invariance kernel $\operatorname{Inv}_{\psi}(D)$ for System (14), $W=\mathbb{R}$

Note that due to Theorem 3.1(iii) ISDS cannot hold because $\operatorname{Inv}_{\psi}(D)$ does not contain an epigraph for any neighborhood $B$ of $A=\{0\}$, i.e., $\mathcal{M}\left(\operatorname{Inv}_{\psi}(D)\right)=\emptyset$. The fact that the system is not ISDS can also be seen directly, because it is easily verified that for $x=0$ and, e.g., $w \equiv 2$ the corresponding trajectory grows unboundedly, it even tends to $\infty$ in finite time.

This gives rise to the question about the meaning of this nontrivial invariance kernel. The answer can be given when looking at the set $W$ of admissible perturbation values. In fact, the shape of the invariance kernel in Figure 1 still contains what could be called a restricted epigraph, i.e., a set of the form $\operatorname{Epi}(V) \cap\left(\mathbb{R}^{n} \times[0, \hat{y}]\right)$ for some function $V$ and some $\hat{y}>0$. It turns out that choosing the "right" $\hat{y}$ with this property, we can prove ISDS for a suitably restricted set $W \subset W$ of perturbation values. In order to make this statement precise and to formulate a necessary and sufficient condition we need the horizontal cross section

$$
\mathcal{S}\left(\operatorname{Inv}_{\psi}(D), y\right):=\left\{x \in \mathbb{R}^{n} \mid(x, y) \in \operatorname{Inv}_{\psi}(D)\right\}
$$

of the set $\operatorname{Inv}_{\psi}(D) \subset \mathbb{R}^{n+1}$ and the invariance kernel of a set $S \subset \mathbb{R}^{n}$ under the solutions $\varphi$ of (4) with perturbations from $W \subset \mathbb{R}^{l}$ defined by

$$
\operatorname{Inv}_{\varphi, W}(S):=\left\{\begin{array}{l|l}
x \in S & \begin{array}{l}
\varphi(t, x, w) \in S \text { for all } \\
w \in L_{\infty}(\mathbb{R}, W), x \in S, t \geq 0
\end{array}
\end{array}\right\} .
$$

Theorem 4.1: Consider a compact set $A \subset \mathbb{R}^{n}$ and the set $\operatorname{Inv}_{\psi}(D)$ for $D$ from (11).

(i) Assume that for some real number $\hat{y}>0$ and the perturbation range $\widetilde{W}:=\{w \in W \mid \gamma(\|w\|) \leq \hat{y}\}$ the set

$$
C=\operatorname{Inv}_{\varphi, \widetilde{W}}\left(\mathcal{S}\left(\operatorname{Inv}_{\psi}(D), \hat{y}\right)\right)
$$


contains a neighborhood $B$ of $A$ for which we can find a $\sigma \in \mathcal{K}_{\infty}$ with the property

$$
\operatorname{Epi}\left(\left.\sigma\left(d_{A}\right)\right|_{B}\right) \cap\left(\mathbb{R}^{n} \times[0, \hat{y}]\right) \subseteq \operatorname{Inv}_{\psi}(D)
$$

Then the set $A$ is ISDS with neighborhood $B$ and perturbation range $\widetilde{W}$.

(ii) Conversely, if the set $A$ is ISDS on some neighborhood $B$ for the perturbation range $\widetilde{W}=\{w \in W \mid \gamma(\|w\|) \leq \hat{y}\}$ for some $\hat{y}>0$, then the assumptions in (i) are satisfied for this value $\hat{y}$ and $C=\mathcal{R}_{\varphi, \widetilde{W}}(B)$.

Proof: (i) We prove the assertion by showing that for the differential inclusion

$$
\begin{aligned}
\dot{x}(t) & \in f(x(t), \widetilde{W}(y(t))) \\
\dot{y}(t) & =-g(y(t)) \\
\text { with } \widetilde{W}(y) & =\{w \in \widetilde{W} \mid \gamma(\|w\|) \leq y\}
\end{aligned}
$$

with solutions denoted by $\tilde{\psi}$ the forward invariance kernel $\operatorname{Inv}_{\tilde{\psi}}(D)$ satisfies (12) for $B$. Then (i) follows from Theorem 3.1(iii).

We prove (12) using the forward invariance of $C$ under $\varphi$ and $\widetilde{W}$. This property implies $\tilde{\psi}_{x}(t, x, \hat{y}) \subset C$ for all $t \geq 0$ and all $x \in C$. In order to show (12), we have to show that for any point $(x, y)$ with $x \in B, y \geq \sigma\left(d_{A}(x)\right)$ and any solution $\tilde{\psi}(t)$ starting from this point the property $\tilde{\psi}(t) \in D$ holds for all $t \geq 0$. In order to accomplish this we show

$$
\begin{aligned}
& \text { there exists } \hat{t} \geq 0 \text { with } \tilde{\psi}(t) \in D \text { for all } t \in[0, \hat{t}] \\
& \text { and } \tilde{\psi}(\hat{t}) \in \operatorname{Inv}_{\psi}(D) \text {. }
\end{aligned}
$$

This will prove (12) since $\operatorname{Inv}_{\psi}(D) \subseteq D$ is forward invariant (16), due to the fact that the solution set of (16) is smaller than that of (10),

If $y \leq \hat{y}$ then (15) implies $(x, y) \in \operatorname{Inv}_{\psi}(D)$, hence (17) holds for $\hat{t}=0$. If $y>\hat{y}$ then we write the solution as $\tilde{\psi}(t)=\left(\tilde{\psi}_{x}(t), \tilde{\psi}_{y}(t)\right)$. Then the forward invariance of $C$ under $\varphi$ carries over to $\tilde{\psi}_{x}$, i.e., $\tilde{\psi}_{x}(t) \in C$ for all $t \geq 0$. Since $\tilde{\psi}_{y}(t) \rightarrow 0$ we obtain $\tilde{\psi}_{y}(\hat{t})=\hat{y}$ for some $\hat{t} \geq 0$ and consequently $\tilde{\psi}(\hat{t}) \in C \subset \operatorname{Inv}_{\psi}(D)$. For $t \in[0, \hat{t}]$ we have $\tilde{\psi}_{y}(t) \geq \hat{y} \geq \sigma\left(d_{A}\left(\tilde{\psi}_{x}(t)\right)\right)$, where the last inequality holds because the point $\left(\tilde{\psi}_{x}(t), \hat{y}\right)$ lies in $C \times\{\hat{y}\} \subseteq \operatorname{Inv}_{\psi}(D) \subseteq$ $D=\operatorname{Epi}\left(d_{A}\right)$. Thus, $\tilde{\psi}(t) \in \operatorname{Epi}\left(d_{A}\right)=D$, which proves (17) in this case.

We have thus shown that $\operatorname{Inv}_{\tilde{\psi}}(D)$ satisfies (12). This finishes the proof of (i) because now the ISDS property follows immediately from Theorem 3.1(iii).

(ii) If ISDS holds for $\widetilde{W}$ on some neighborhood $B$ of $A$, then for this set of perturbations there exists an ISDS Lyapunov function $V: \mathcal{R}_{\varphi, \widetilde{W}}(B) \rightarrow \mathbb{R}$ whose epigraph by Theorem 3.1(i) satisfies $\operatorname{Epi}(V) \subseteq \operatorname{Inv}_{\psi}(D)$ and $\operatorname{Epi}\left(\left.\sigma\left(d_{A}\right)\right|_{B}\right) \subseteq \operatorname{Epi}(V)$ for some $\sigma \in \mathcal{K}_{\infty}$. Since $\mathcal{R}(B) \subseteq$ $S\left(\operatorname{Inv}_{\tilde{\psi}}(D), \hat{y}\right)$ holds, the invariance $\operatorname{kernel}_{\tilde{\psi}}(D)$ satisfies the assumptions from part (i). We have to show that $\operatorname{Inv}_{\psi}(D)$ also satisfies this assumptions, which we do by showing that these sets coincide for $y \leq \hat{y}$. To this end consider the perturbation range $W \supseteq \widetilde{W}$. Then for any point $(x, y)$ with $y \leq \hat{y}$ the set of possible solutions of (10) coincides with that of (16), because we have $W\left(\psi_{y}(t)\right) \subseteq \widetilde{W}$ for all $t \geq 0$. Hence we have

$$
\operatorname{Inv}_{\tilde{\psi}}(D) \cap\left(\mathbb{R}^{n} \times[0, \hat{y}]\right)=\operatorname{Inv}_{\psi}(D)
$$

which shows that the assumptions from (i) also hold for $\operatorname{Inv}_{\psi}(D)$.

Remark 4.2: The equivalence of ISDS with $\widetilde{W}$ and the condition in Theorem 4.1(i) implies that the maximal $\hat{y}$ satisfying this condition characterizes the maximal set of perturbations for which ISDS holds for the considered comparison functions $\gamma$ and $\mu$.

Unfortunately, the first condition of Theorem 4.1(i), i.e., the assumption on the invariance kernel $\operatorname{Inv}_{\varphi, \widetilde{W}}\left(\mathcal{S}\left(\operatorname{Inv}_{\psi}(D), \hat{y}\right)\right)$ is not directly related to the shape of the invariance kernel $\operatorname{Inv}_{\psi}(D)$, hence just by looking at $\operatorname{Inv}_{\psi}(D)$ it is not possible to verify the assumptions of Theorem 4.1(i).

Fortunately, there is a remedy to this problem if one aims at a sufficient ISDS condition analogous to Corollary 3.3. This corollary can be extended to the $\hat{y}$-restricted case without making assumptions on $\operatorname{Inv}_{\varphi, \widetilde{W}}\left(\mathcal{S}\left(\operatorname{Inv}_{\psi}(D), \hat{y}\right)\right)$. The key observation for this result is the following lemma, which gives a sufficient condition for the forward invariance of the set $\mathcal{S}\left(\operatorname{Inv}_{\psi}(D), \hat{y}\right)$ itself under $\varphi$.

Lemma 4.3: Assume that there exists $\varepsilon>0$ such that the condition

$$
\mathcal{S}\left(\operatorname{Inv}_{\psi}(D), y\right) \subseteq \mathcal{S}\left(\operatorname{Inv}_{\psi}(D), \hat{y}\right)
$$

holds for all $y \in(\hat{y}-\varepsilon, \hat{y})$ and some $\hat{y}>0$. Then

$$
\operatorname{Inv}_{\varphi, \widetilde{W}}\left(\mathcal{S}\left(\operatorname{Inv}_{\psi}(D), \hat{y}\right)\right)=\mathcal{S}\left(\operatorname{Inv}_{\psi}(D), \hat{y}\right)
$$

for the perturbation range $\widetilde{W}=\{w \in W \mid \gamma(\|w\|) \leq \hat{y}\}$.

Proof: We abbreviate $C:=\mathcal{S}\left(\operatorname{Inv}_{\psi}(D), \hat{y}\right)$ and show that $C$ is forward invariant for all perturbation functions $w \in \mathcal{W}$ with $\alpha:=\gamma\left(\|w\|_{\infty}\right)<\hat{y}$. By continuity this implies the desired result also for $\alpha=\hat{y}$.

Consider a point $x \in C$ and a perturbation function $w \in \widetilde{\mathcal{W}}$ with $\alpha<\hat{y}$. We prove the forward invariance by contradiction. For this purpose assume that there exists a time $t>0$ such that $\varphi(t, x, w) \notin C$. Consider a time $\Delta t>0$ with the property that $\mu(\hat{y}, \Delta t)>\max \{\alpha, \hat{y}-\varepsilon\}$, which exists by continuity of $\mu$ and since $\hat{y}>\alpha$. Since $\varphi$ starts in $C$ we find a time $t_{1} \geq 0$ with

$$
\varphi\left(t_{1}, x, w\right) \in C \text { and } \varphi\left(t_{1}+\Delta t, x, w\right) \notin C .
$$

From the choice of $\Delta t$ we obtain $\|w(t)\| \leq \mu\left(y^{*}, t\right)$ for almost all $t \in\left[0, t_{1}+\Delta t\right]$. Hence, for $t \in\left[t_{1}, t_{1}+\Delta t\right]$ the function $\psi(t)=\left(\varphi(t, x, w), \mu\left(y^{*}, t\right)\right)$ is a solution of the differential inclusion (10). Furthermore, by the definition of $y^{*}$ the point $\left(\varphi\left(t_{1}, x, w\right), \hat{y}\right)$ lies in $\operatorname{Inv}_{\psi}(D)$. Thus, the forward invariance of $\operatorname{Inv}_{\psi}(D)$ implies $\psi\left(t_{1}+\Delta t\right) \in \operatorname{Inv}_{\psi}(D)$ which in particular yields $\varphi\left(t_{1}+\Delta t, x, w\right) \in \mathcal{S}\left(\operatorname{Inv}_{\psi}(D), \mu(\hat{y}, \Delta t)\right.$ $\subseteq C$ which contradicts the choice of $t_{1}$ and $\Delta t$. Thus $C$ is forward invariant under $\varphi$.

Using this fact we can state the following result, which is analogous to Corollary 3.3. 
Corollary 4.4: Consider the perturbed system (4) and the differential inclusion (10). Consider a compact set $A \subset \mathbb{R}^{n}$, an open neighborhood $B \subset \mathbb{R}^{n}$ of $A$ and the set $D$ from (11).

Assume that there exists a function $V: \mathbb{R}^{n} \rightarrow \mathbb{R} \cup \infty$, a function $\sigma \in \mathcal{K}_{\infty}$ and a value $\hat{y}>0$ such that

$$
\begin{aligned}
\operatorname{Epi}\left(\left.\sigma\left(d_{A}\right)\right|_{B}\right) \cap\left(\mathbb{R}^{n} \times[0, \hat{y}]\right) & \subseteq \operatorname{Epi}(V) \cap\left(\mathbb{R}^{n} \times[0, \hat{y}]\right) \\
& =\operatorname{Inv}_{\psi}(D) \cap\left(\mathbb{R}^{n} \times[0, \hat{y}]\right)
\end{aligned}
$$

holds. Then $V$ is an ISDS Lyapunov function on $B$ for the perturbation range $\widetilde{W}=\{w \in W \mid \gamma(\|w\| \leq \hat{y}\}$. In particular, the set $A$ is ISDS with neighborhood $B$ for perturbation range $\widetilde{W}$.

Proof: From $\operatorname{Epi}(V) \cap\left(\mathbb{R}^{n} \times[0, \hat{y}]\right)=\operatorname{Inv}_{\psi}(D) \cap\left(\mathbb{R}^{n} \times[0, \hat{y}]\right)$ we obtain the equality

$$
\mathcal{S}\left(\operatorname{Inv}_{\psi}(D), y\right)=V^{-1}([0, y])
$$

for all $y \in[0, \hat{y}]$. This immediately implies $\mathcal{S}\left(\operatorname{Inv}_{\psi}(D), y_{1}\right) \subseteq \mathcal{S}\left(\operatorname{Inv}_{\psi}(D), y_{2}\right)$ if $0 \leq y_{1} \leq y_{2} \leq \hat{y}$ hence by Lemma 4.3 we obtain $\operatorname{Inv}_{\varphi, \widetilde{W}}\left(\mathcal{S}\left(\operatorname{Inv}_{\psi}(D), \hat{y}\right)\right)=$ $\mathcal{S}\left(\operatorname{Inv}_{\psi}(D), \hat{y}\right)$. Thus, Theorem 4.1 (i) yields the assertion.

We can apply this result to our Example (14) with $\operatorname{Inv}_{\psi}(D)$ from Figure 1. There one sees that the condition of Corollary 4.4 is satisfies e.g. for $\hat{y}=0.24$. Note that for large $\hat{y}$ the assumed epigraph property from Corollary 4.4 is not satisfied and the inclusion $\mathcal{S}\left(\operatorname{Inv}_{\psi}(D), y\right) \subseteq \mathcal{S}\left(\operatorname{Inv}_{\psi}(D), \hat{y}\right)$ for $y \leq \hat{y}$ does not hold. Since $\gamma(r)=2 r$, we obtain ISDS with $\widetilde{W}=[-0.12,0.12]$. The numerical computation of the corresponding invariance kernel $\operatorname{Inv}_{\tilde{\psi}}(D)$ as shown in Figure 2 indicates that this is the case because now the invariance kernel is indeed an epigraph.

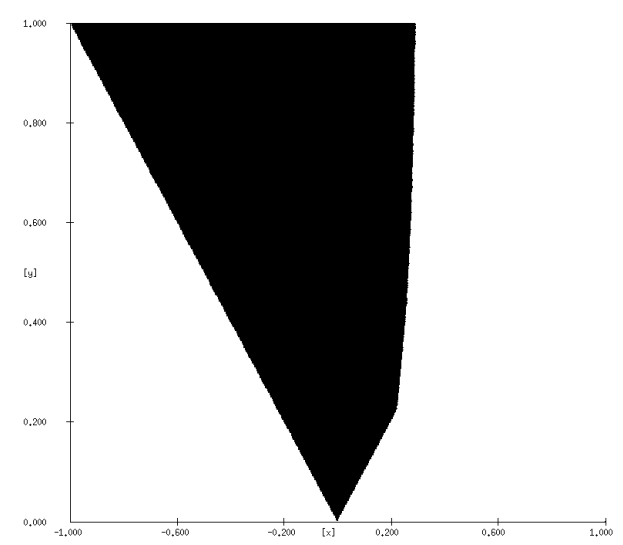

Fig. 2. Numerically determined invariance kernel $\operatorname{Inv}_{\tilde{\psi}}(D)$ for System (14), $\widetilde{W}=[-0.12,0.12]$

\section{EXAMPLES}

Invariance Kernels and their boundaries can be computed by set valued numerical techniques, using the algorithms developed in [11] and extended in [2]. Our approach therefore allows to compute ISDS Lyapunov functions numerically. In this section we provide two examples which were computed with these algorithms and illustrate our theoretical results.

The first example is motivated by a question which arises when looking at our results: is it possible that $\operatorname{Inv}_{\psi}(D)$ contains a "maximal" epigraph $\operatorname{Epi}(V)=\mathcal{M}\left(\operatorname{Inv}_{\psi}(D)\right)$ but is not equal to this set, i.e., $\emptyset \neq \mathcal{M}\left(\operatorname{Inv}_{\psi}(D)\right) \neq \operatorname{Inv}_{\psi}(D)$ ?

Indeed, this situation is possible, as the one dimensional example

$$
\dot{x}(t)=-2 x(t)(1 / 2-x)^{2}+(1 / 4+x(t))^{2} w(t)
$$

shows. Figure 3 shows the numerically determined invariance kernel for $\gamma(r)=r / 2$ and $g(r)=r / 10$.

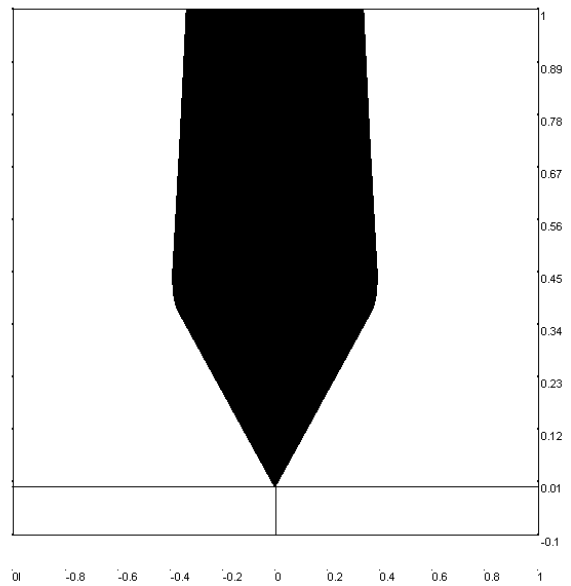

Fig. 3. Numerically determined invariance $\operatorname{kernel}^{\operatorname{Inv}_{\tilde{\psi}}}(D)$ for System (18), $\widetilde{W}=\mathbb{R}$

Here one observes that $\operatorname{Inv}_{\psi}(D)$ contains the epigraph of the function $V(x)=|x|$ for $x \in[-1 / 4,1 / 4]$ but, in addition, also a restricted epigraph of the same function on a larger interval.

The reason for this behavior is due to the fact that the system is ISDS for unrestricted perturbation on $B=$ $[-1 / 4,1 / 4]$ because the perturbation cannot drive the system out of this set. For smaller perturbations, however, it is ISDS on larger sets which is why $\left.\operatorname{Inv}_{\psi}(D)\right)$ contains additional points.

The second example is a two dimensional system which is easily verified to be ISS (hence ISDS) because it is a cascade of two ISS systems. It is given by

$$
\begin{aligned}
& \dot{x}_{1}(t)=-x_{1}(t)+3 x_{2}(t) \\
& \dot{x}_{2}(t)=-x_{2}(t)+w(t)
\end{aligned}
$$

For $\gamma(r)=10 r$ and $g(r)=r / 10$ Figure 4 (left) shows the lower boundary of the invariance kernel, which in this case happens to be an epigraph, i.e., the figure shows the graph of the ISDS Lyapunov function which was computed using the functional approximation. Figure 4 (right) shows the corresponding level sets. 

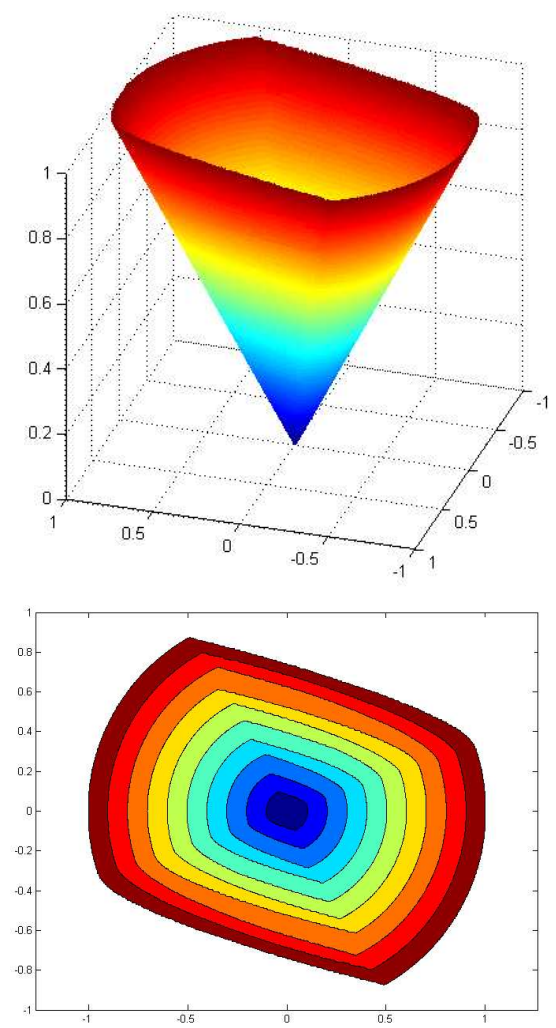

Fig. 4. Graph and contour sets for the ISDS Lyapunov function for System (19), $\widetilde{W}=\mathbb{R}$

\section{CONCLUSIONS}

The shape of the contour set in our last example suggests that the minimal ISDS Lyapunov function is nonsmooth, indicating that optimal ISDS Lyapunov functions are not in general smooth, a property which is also known for optimal $H_{\infty}$ storage functions, see [10]. Indeed, since the epigraph of the minimal ISDS Lyapunov function is an invariance kernel and since the invariance kernel is a maximal closed subset (satisfying the invariance property), the minimal ISDS Lyapunov function is necessarily lower semicontinuous but in general it has no reason to be smooth or even continuous. This motivates our use of set oriented methods and setvalued analysis, which is an appropriate framework for handling such functions.

\section{REFERENCES}

[1] J.-P. Aubin and A. Cellina, Differential Inclusions. Springer-Verlag, 1984.

[2] P. Cardaliaguet, M. Quincampoix, and P. Saint-Pierre, "Set-valued numerical analysis for optimal control and differential games," in Stochastic and differential games, ser. Ann. Internat. Soc. Dynam. Games. Boston, MA: Birkhäuser, 1999, vol. 4, pp. 177-247.

[3] F. Colonius and W. Kliemann, "Limits of input-to-state stability," Syst. Control Lett., vol. 49, no. 2, pp. 111-120, 2003.

[4] L. Grüne, Asymptotic Behavior of Dynamical and Control Systems under Perturbation and Discretization, ser. Lecture Notes in Mathematics, Vol. 1783. Springer-Verlag, 2002.

[5] _ - "Input-to-state dynamical stability and its Lyapunov function characterization," IEEE Trans. Autom. Control, vol. 47, pp. 14991504, 2002.

[6] — - "Quantitative aspects of the input-to-state stability property," in Optimal Control, Stabilization, and Nonsmooth Analysis, ser. Lecture Notes in Control and Information Sciences 301, M. de Queiroz, M. Malisoff, and P. Wolenski, Eds. Springer-Verlag, Heidelberg, 2004, pp. 215-230.

[7] L. Grüne, E. D. Sontag, and F. R. Wirth, "Asymptotic stability equals exponential stability, and ISS equals finite energy gain-if you twist your eyes," Syst. Control Lett., vol. 38, pp. 127-134, 1999.

[8] S. Huang, M. James, D. Nesic, and P. M. Dower, "Analysis of input to state stability for discrete time nonlinear systems via dynamic programming," in Proceedings of the 42th IEEE Conference on Decision and Control, Hawaii, USA, 2003, pp. 5068-5073, full version provisionally accepted in Automatica.

[9] E. B. Lee and L. Markus, Foundations of Optimal Control. John Wiley \& Sons, New York, 1967.

[10] L. Rosier and E. D. Sontag, "Remarks regarding the gap between continuous, Lipschitz, and differentiable storage functions for dissipation inequalities," Syst. Control Lett., vol. 41, pp. 237-249, 2000.

[11] P. Saint-Pierre, "Approximation of the viability kernel," Appl. Math. Optim., vol. 29, pp. 187-209, 1994.

[12] E. D. Sontag, "Smooth stabilization implies coprime factorization," IEEE Trans. Autom. Control, vol. 34, pp. 435-443, 1989.

[13] — - "On the input-to-state stability property," Europ. J. Control, vol. 1, pp. 24-36, 1995.

[14] — - "The ISS philosophy as a unifying framework for stabilitylike behavior," in Nonlinear Control in the Year 2000, Volume 2, ser. Lecture Notes in Control and Information Sciences 259, A. Isidori, F. Lamnabhi-Lagarrigue, and W. Respondek, Eds., NCN. Springer Verlag, London, 2000, pp. 443-468.

[15] E. D. Sontag and Y. Wang, "On characterizations of the input-to-state stability property," Syst. Control Lett., vol. 24, pp. 351-359, 1995. 